\title{
BMJ Open Treatment of hypertension reduces cognitive decline in older adults: a systematic review and meta-analysis
}

\author{
Aditi Gupta (D) , ${ }^{1,2}$ Sophy Perdomo, ${ }^{3}$ Sandra Billinger, ${ }^{3,4}$ Srinivasan Beddhu, ${ }^{5}$ \\ Jeffrey Burns, ${ }^{2,4}$ Gary Gronseth ${ }^{4}$
}

To cite: Gupta A, Perdomo S, Billinger S, et al. Treatment of hypertension reduces cognitive decline in older adults: a systematic review and meta-analysis. BMJ Open 2020;10:e038971. doi:10.1136/ bmjopen-2020-038971

- Prepublication history and supplemental material for this paper are available online. To view these files, please visit the journal online (http://dx.doi. org/10.1136/bmjopen-2020038971).

Received 31 March 2020 Revised 27 July 2020

Accepted 17 October 2020

Check for updates

(c) Author(s) (or their employer(s)) 2020. Re-use permitted under CC BY-NC. No commercial re-use. See rights and permissions. Published by BMJ.

${ }^{1}$ Division of Nephrology and Hypertension, Department of Internal Medicine, University of Kansas Medical Center, Kansas City, Kansas, USA

${ }^{2}$ Alzheimer's Disease Center, University of Kansas Medical Center, Kansas City, Kansas, USA ${ }^{3}$ Department of Physical Therapy and Rehabilitation Science, University of Kansas Medical Center, Kansas City, Kansas, USA ${ }^{4}$ Department of Neurology, University of Kansas Medical Center, Kansas City, Kansas, USA

${ }^{5}$ Division of Nephrology and Hypertension, University of Utah, Salt Lake City, Utah, USA

Correspondence to

Dr Aditi Gupta;

agupta@kumc.edu

\section{ABSTRACT}

Objectives To systematically analyse the effect of pharmacological treatment of hypertension (HTN) on cognitive decline in older adults.

Methods Randomised, placebo-controlled trials with a prespecified quantitative outcome of cognition and a pharmacological intervention for at least 12 months to treat HTN in older adults ( $>60$ years). Our primary outcome was change in cognition with pharmacological treatment of HTN. Standardised mean difference (SMD) was used to analyse different outcomes reported in the selected studies. We searched PubMed CENTRAL and the Cochrane Library from inception to 6 July 2020. Two independent reviewers assessed trial quality and extracted data. Internal and external validity of the studies was assessed. Results Nine randomised controlled trials with 34994 participants were included in the final analysis. The net SMD for change in cognition was -0.049 ( $\mathrm{Cl}$ : -0.078 to -0.019 ) indicating that treatment of HTN decreased cognitive decline. Heterogeneity was low with an $\mathrm{I}^{2}$ of $6 \%$. Discussion Current evidence does not indicate worsening of cognition with treatment of HTN. Treatment of HTN in older adults may reduce cognitive decline. These results have important implications in clinical management of patients at risk for dementia.

PROSPERO registration number CRD42020139750.

\section{INTRODUCTION}

Dementia is a disease of the old with an estimated prevalence of 45 million worldwide. ${ }^{1}$ With increasing life expectancy and an upsurge in the ageing population, these numbers are expected to rise and the number of persons with dementia is projected to triple by $2050 .^{2}$ Currently, effective pharmacological treatment for dementia is limited. Approximately half of Alzheimer's disease cases are attributable to modifiable risk factors and even a modest $10 \%-25 \%$ reduction in these risk factors could cut the number of adults in the USA with Alzheimer's by nearly 500 000 cases. $^{3}$ Thus, non-pharmacological interventions such as aggressive management of hypertension (HTN) are being investigated.

HTN affects an estimated 1.39 billion people worldwide, ${ }^{4}$ and up to two-thirds of

\section{Strengths and limitations of this study}

- This is a meta-analysis of nine published randomised controlled trials with almost 35000 participants included in this analysis.

- We converted ORs to standardised mean difference, which enabled us to include studies reporting incident dementia, mild cognitive impairment or cognitive decline on continuous measures.

- To improve the clinical interpretability of the results, we estimated the absolute magnitude of effect of aggressive treatment of hypertension on reducing cognitive decline.

- The included studies were heterogeneous in blood pressure goals, antihypertensives used and acquired blood pressure at the end of the study.

- Since this analysis includes randomised clinical trials, results may not be generalisable to the old and frail patients who may not participate in clinical trials.

people $>65$ years of age. HTN is an independent risk factor for dementia and Alzheimer's disease. ${ }^{5-8}$ Longstanding HTN leads to vascular remodelling, decrease in vascular compliance and increase in pulse pressure ${ }^{9}$ decrease in cerebral blood flow, ${ }^{10}$ degenerative changes of the vessel wall ${ }^{11}$ and decrease in cerebrovascular reserve. ${ }^{12}$ These changes disrupt cerebral autoregulation and lead to increased white matter lesions, ${ }^{13}$ microinfarcts, microhaemorrhages and cerebrovascular events, ${ }^{14}$ known causes for faster cognitive decline and incident dementia. ${ }^{1516}$

Despite these associations and other known adverse effects of $\mathrm{HTN}^{14}$ blood pressure (BP) remains inadequately controlled in three out of four older adults ( $>65$ years) with HTN. ${ }^{17} 18$ Concerns about decreasing cerebral perfusion and thereby worsening cognition with lowering of systemic BP in older adults have limited aggressive HTN management in clinical practice. ${ }^{19-22}$ However, studies indicate protective adaptation of the 
cerebral vasculature with BP reduction to prevent cerebral hypoperfusion. ${ }^{23} 24$

The objective of this meta-analysis is to systematically analyse the effect of pharmacological treatment of HTN on cognitive decline in older adults. Previous systematic reviews and meta-analyses have reported conflicting and equivocal results on the effect of treating HTN on cognition. ${ }^{25-28}$ In the absence of conclusive evidence regarding safety of BP reduction in older persons at risk of dementia, clinical decision-making remains challenging. Since the publication of the last systematic review on treatment of HTN and cognition in $2009,{ }^{28}$ new studies evaluating the effect of treating HTN on cognitive decline and dementia have been published. Here, we present the results of a systematic review and meta-analysis of existing randomised controlled clinical trials on the effect of pharmacological treatment of HTN on cognition in older persons without previous stroke.

\section{METHODS}

We conducted a systematic review and meta-analysis to assess the effect of treatment of HTN on cognition.

\section{Inclusion criteria}

Studies were included in the final analysis if they were (1) randomised controlled trials on pharmacological treatment of HTN, (2) in adult participants where the majority of participants were $>60$ years old, (3) with a pre-specified outcome of cognition and with a measure of cognition such as a standard neuropsychological test (eg, Mini-Mental State Examination (MMSE)), (4) with comparison group with either placebo, no intervention, standard of care or a higher BP goal and (5) with at least 1-year follow-up. The randomisation could be for a BP goal or by medications for HTN. Observational studies and studies specifically assessing treatment of HTN after stroke were excluded. However, studies where stroke was not listed as a specific inclusion criterion (ie, some but not all participants may have a history of stroke) were included.

\section{Changes from PROSPERO protocol}

We made some modifications to the published protocol to address unanticipated characteristics of the available studies. These changes were made after our initial review of the identified papers but before extraction of study results.

1. The initial intended target age was people $>60$ years of age. However, only five studies published prior 2008 met this criterion. Therefore, we modified this selection criterion to allow inclusion of all studies of adults where the median or mean age of participants was $\geq 60$ years.

2. Several studies identified in the search exclusively enrolled patients with stroke or transient ischaemic attack. Since we were not primarily interested in the effect of aggressive BP control on recurrent stroke (which could affect cognitive function), we excluded these studies. We included studies enrolling some patients with stroke as long as they constituted a minority of patients.

\section{Data sources and searches}

We searched PubMed CENTRAL and the Cochrane Library (from inception to 6 July 2020) for relevant studies (online supplemental data A). Search included keywords such as cognition, dementia or cognitive dysfunction, HTN or antihypertensive agents, placebo or control and randomised clinical trial.

\section{Patient and public involvement statement}

Individual patients were not recruited for this study or involved in the study in any other way. The study was inspired by questions arising during clinical care of older patients. Dementia is a public health crisis and discovering ways to reduce cognitive decline is important for patients. Considering the high prevalence of HTN in the old, even a small effect of BP reduction on cognitive decline is clinically relevant.

\section{Study selection}

Two independent reviewers (AG and SP) reviewed titles and abstracts to select articles for further review. In case of disagreements, the manuscript in question was included with an intention of being more inclusive at this stage. After assessment of inclusion and exclusion criteria, the qualifying studies were selected for the final analysis. Two reviewers independently abstracted data elements from the selected studies. Disagreements between the two reviewers were resolved with mutual discussion and in some cases by involving a third member (GG).

\section{Data extraction and quality assessment}

We developed a data extraction form in Microsoft Excel. For internal validity assessment, this form included fields regarding Cochrane risk of bias domains for therapeutic studies. For external validity assessment, we gathered information on about baseline characteristics, inclusion and exclusion criteria, enrolment, study setting, study methods, intervention for treatment of HTN, BP goals, change in $\mathrm{BP}$, achieved $\mathrm{BP}$, difference in $\mathrm{BP}$ in the intervention versus control groups, study size, outcomes, effect, primary and secondary outcomes for cognition and study duration. To ensure consistency in data extraction, we piloted the form using studies that were excluded from this analysis. Two reviewers independently extracted the data listed in this form from the selected studies. A third reviewer $(\mathrm{GG})$ spot checked certain studies for data extraction for accuracy.

The primary measure for assessing cognition was different in the selected studies. Continuous measures of cognition were extracted where available. The mean change in the primary measure for cognition, the SD and the number of participants in the intervention and control groups was extracted. In the absence of a continuous measure of cognition, dichotomous measures or ORs were extracted. For the 
selected studies, the number of participants in each treatment group and the number experiencing the outcome selected were extracted. When available, results from an intention-to-treat analysis were chosen.

\section{Data synthesis and analysis}

To maximise precision, our preferred outcome measure was the difference in the change in a continuous measure of global cognition from baseline. Because we anticipated that change in cognition would be measured on different continuous scales, we used standardised mean differences (SMDs) as our primary effect size measure. For studies reporting the count of events (eg, the number of participants developing dementia) and not continuous cognitive measures, we converted ORs to SMDs using the Hasselblad and Hedges method. ${ }^{29}{ }^{30}$ We calculated 95\% CIs of the SMDs as our measure of precision. We used inverse variance, random effects model to calculate a summary measure of effect and $\mathrm{I}^{2}$ as the measure of heterogeneity.

We performed a cumulative meta-analysis according to the date of publication to detect overall estimate changes over time. We performed exploratory random effects meta-regression to examine the impact of moderator variables for change in systolic BP (SBP), baseline SBP, year of publication, participant age, duration of follow-up, prevalence of diabetes mellitus and proportion of women in the study on the relationship between cognition and treatment of HTN. We assessed publication bias by inspecting a funnel plot of SE by effect size and with Egger's regression test. ${ }^{31}$ We calculated the absolute effect of treatment of HTN on cognition and number needed to treat (NNT) from studies reporting ORs or dichotomous outcomes.

\section{Sensitivity analysis}

For sensitivity analysis, we assessed the impact of individual studies on the summary effect by performing a one-study-removed analysis. To determine whether the results of our analysis were affected by the choice of effect size measure (continuous vs binary), we separately meta-analysed studies selecting a continuous measure of cognition (where we converted a raw mean difference to an SMD) from those selecting a binary measure of cognition (where we converted an OR to an SMD). We also did a fixed effect meta-analysis. Additionally, to determine if modifications to the analysis plan influenced the results, we meta-analysed studies that only met our original protocol's age criteria (no patients $\leq 60$ years old).

Analysis was done using Comprehensive Meta-Analysis V. $3^{32}$ and Microsoft Excel.

\section{RESULTS}

We identified 2072 records of interest with our search strategy (figure 1) and reviewed 1863 abstracts after removing duplicate records. Of these, 28 full-text articles from 14 independent trials were selected for further review. Final analysis included nine studies. ${ }^{33-41}$ The reasons for exclusion of remaining 19 studies are listed in online supplemental data B. A Preferred Reporting Items for Systematic Reviews and Meta-Analyses flow diagram of the selection of included studies is presented in figure 1 . We included three studies with participants $>55$ years old $^{37} 3940$ and a study with participants $>50$ years old, ${ }^{41}$ as they also included participants $>60$ years old as outlined in our inclusion criteria.

\section{Assessment of bias in included studies Internal validity}

All included studies had adequate sequence generation except the Systolic Hypertension in the Elderly Program (SHEP) ${ }^{33}$ where the method of sequence generation was unclear. Allocation concealment was unclear in Medical Research Council (MRC) treatment trial of HTN by Prince et $a l^{34}$ and the study by Pantoni $e t a l .^{37}$ Characteristics of prognostic importance were substantially similar between treatment groups in all studies except SHEP, ${ }^{33}$ where the data presented were inadequate to assess balance in baseline characteristics (figure 2 and online supplemental data table 1 ). The study by Pantoni $e t a \hat{l}^{37}$ was described as double blinded; however, it was unclear if the outcome assessor was also blinded. MRC, ${ }^{34}$ Memory in Diabetes (MIND) substudy of the Action to Control Cardiovascular Risk in Diabetes (ACCORD) ${ }^{40}$ and the Systolic Blood Pressure Intervention Trial (SPRINT) MIND $^{41}$ were open label studies. All studies had a predefined cognitive outcome and specific tests included in the protocol for assessment of cognition. Attrition was low in all studies.

\section{External validity}

Table 1 shows important characteristics of the included studies. All included studies ${ }^{33-41}$ were multicentre studies, including both men and women. Mean follow-up was up to 5 years.

\section{Participants}

The participants in SHEP, ${ }^{33}$ MRC, ${ }^{34}$ Systolic Hypertension in Europe ${ }^{35}$ the Study on Cognition and Prognosis in the Elderly (SCOPE), ${ }^{36}$ study by Pantoni $e t a l^{37}$ and the SPRINT $^{41}$ had similar ages, whereas participants in the Hypertension in the Very Elderly Trial ${ }^{38}$ were older, and those in TRANSCEND (Telmisartan Randomized Assessment Study in ACE Intolerant Subjects with Cardiovascular Disease) ${ }^{39}$ and the ACCORD-MIND ${ }^{40}$ were younger. All selected studies except the one by Pantoni et $a l^{37}$ either excluded or had a small proportion of participants with known dementia. Insulin use or a history of diabetes was an exclusion criterion for most studies except TRANSCEND, ${ }^{39}$ where $36 \%$ participants had diabetes, and ACCORD,${ }^{40}$ where all participants had diabetes.

\section{Intervention}

The pharmacological therapies and attained BP varied across the studies. BP goals were different in the selected studies with higher BP goal (SBP $<160 \mathrm{~mm} \mathrm{Hg}$ or $150 \mathrm{~mm}$ $\mathrm{Hg}$ ) in older studies, ${ }^{33-36} 38$ and lower BP goals (SBP $<120$ $\mathrm{mm} \mathrm{Hg}$ ) in more recent studies. ${ }^{40}$ Some studies did not 


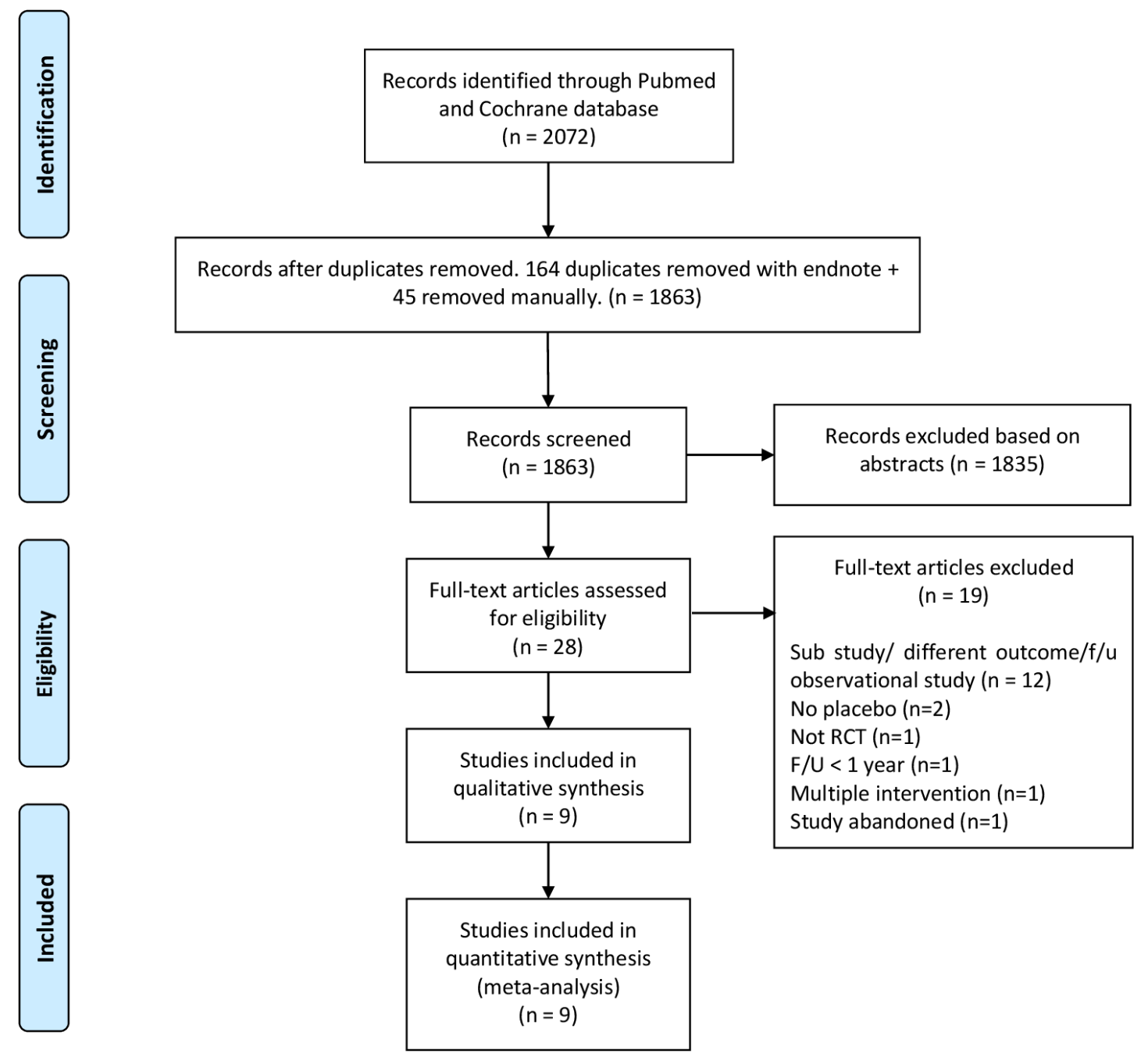

Figure 1 A Preferred Reporting Items for Systematic Reviews and Meta-Analyses diagram.

specify a BP goal. ${ }^{37}{ }^{39}$ The study by Pantoni $e t a l^{37}$ did not show any change in SBP compared with baseline.

\section{Outcome measures}

We used continuous measures of cognition where possible such as MMSE ${ }^{36}{ }^{37}$ Short-Comprehensive Assessment and Referral Evaluation (CARE) cognitive impairment, ${ }^{33}$ paired associate learning test score ${ }^{34}$ and Digit Symbol Substitution Test. ${ }^{40}$ We used cognitive decline, ${ }^{38}{ }^{39}$ incident dementia ${ }^{35}$ or adjudicated mild cognitive impairment ${ }^{41}$ in others where a continuous measure was not available for analysis.

We judged the risk of bias to be low to moderate in the studies. All nine studies were included in the quantitative synthesis.

\section{Effect sizes}

Figure 3 lists the effect sizes and accompanying forest plot for differences in the change in cognition (SMDs with $95 \% \mathrm{CI}$ ) for the included studies. The pooled SMD for change in cognition was -0.049 (95\% CI: -0.078 to $-0.019)$ indicating a positive effect on cognition with treatment of HTN. $\mathrm{I}^{2}$ was $6 \%$ indicating low heterogeneity. The fixed effect meta-analysis was very similar (SMD
$-0.048,95 \%$ CI: -0.077 to -0.020$)$ to the random effects given the low heterogeneity.

Pooled results from studies with a continuous measure of cognition 33343740 (SMD -0.045, 95\% CI: -0.087 to -0.004 ) were substantially similar to those with a binary measure of cognition ${ }^{35} 36383941$ (SMD -0.056, 95\% CI: -0.112 to 0 ) (online supplemental data figure 1 ). $P$ value for interaction was 0.76 .

Cumulative meta-analysis for detection of temporal trends indicated significant point estimates at all but two time points (1996 and 1998) (online supplemental data figure 2). Random effects meta-analysis with one study removed at a time did not substantially change the summary effect (not shown). A meta-analysis restricted to studies enrolling patients over 60 years of age alone also demonstrated substantially similar results (SMD $-0.047,95 \%$ CI: -0.084 to $-0.009)$. Random effects meta-regression for change in SBP, baseline SBP, year of publication, participant age, duration of follow-up, prevalence of diabetes mellitus and proportion of women in the study did not change the effect on cognition (online supplemental data table 2).

The funnel plot used to assess publication bias is shown in online supplemental data figure 3. Egger's regression 


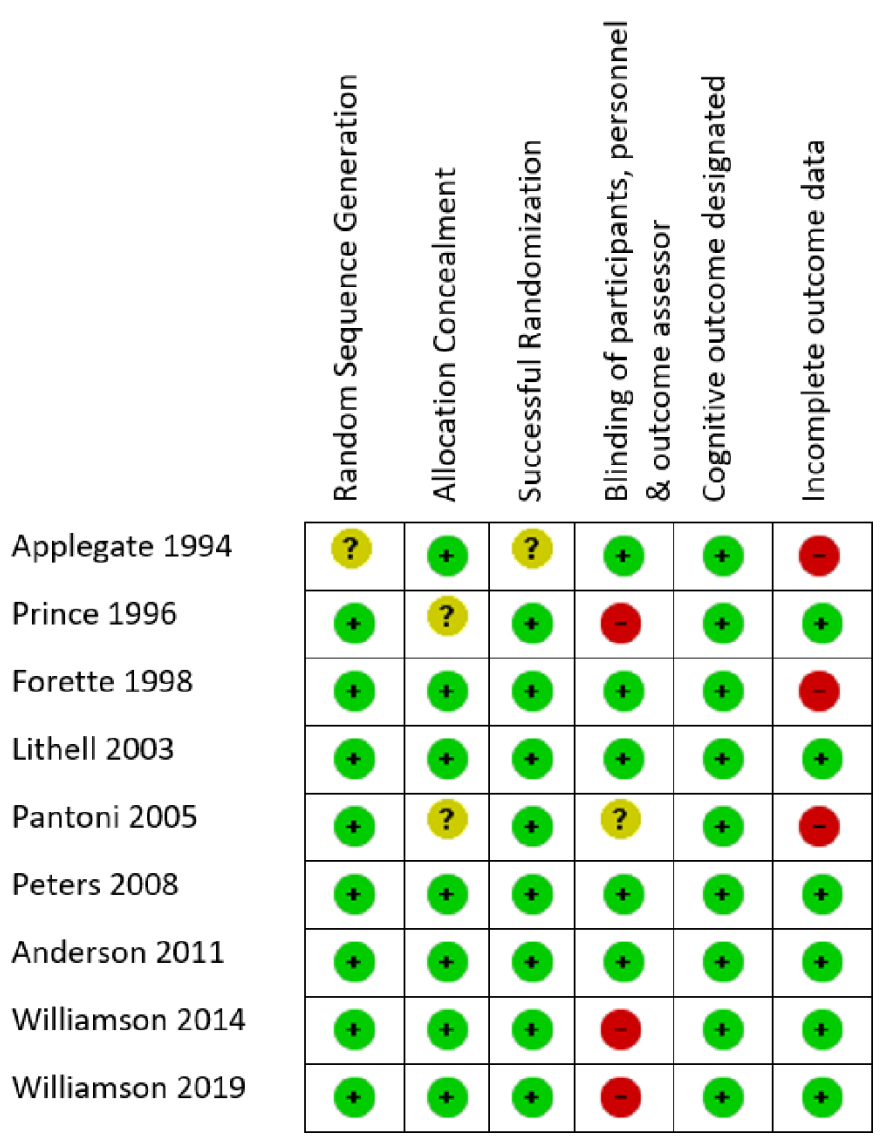

Figure 2 Risk of bias assessment: green indicates low risk of bias; yellow indicates medium risk of bias and red indicates high risk of bias.

test demonstrated no significant association between $\mathrm{SE}$ and effect size $(\mathrm{p}=0.54)$. However, inspection of the plot suggested asymmetry. Thus, we adjusted for potential publication bias with a trim and fill procedure. ${ }^{42}$ The estimated effect size was not substantially changed by this adjustment (SMD $-0.047,95 \%$ CI: -0.081 to -0.014 ).

\section{DISCUSSION}

Our analysis of the current evidence from randomised trials indicates that pharmacological treatment of HTN in older adults without prior stroke modestly reduces cognitive decline (figure 2). Contrary to previous fears, treatment of HTN did not worsen cognitive decline. The reduction in cognitive decline with treatment of HTN was consistent with variable SBP goals, although the study with the largest effect had a more aggressive SBP goal of $120 \mathrm{~mm} \mathrm{Hg} .{ }^{41}$ The benefit of reducing cognitive decline was also consistent over time from 1994 to 2020 when the studies included in this meta-analysis were published. The benefit of treatment of HTN on cognition persisted after adjusting for the impact of moderator variables of vascular dementia and diabetes in the random effects meta-regression (online supplemental data table 2).

Previous reviews and meta-analysis found equivocal results. $^{28} 4344$ A Cochrane review by McGuinness et $a l^{28}$ included four studies and did not report a benefit in cognition with treatment of HTN. New studies ${ }^{39-41}$ with lower SBP goals ${ }^{41}$ have been published since. More recent reviews indicated benefit of treatment of HTN in prospective cohort studies, ${ }^{43}{ }^{45}$ but not in randomised controlled trials. ${ }^{43}$ No harm in cognition or rates of incident dementia were observed with lowering BP. Our analysis shows a small beneficial effect on cognition, perhaps due to increased precision due to inclusion of a larger number of studies. We converted ORs to SMD, which enabled us to include studies reporting incident dementia, mild cognitive impairment or cognitive decline on continuous measures. A recently published review $^{44}$ showed a lower risk of incident dementia or cognitive impairment similar to our study. However, both studies included different populations; with a goal to assess primary prevention with treatment of HTN, and to decrease heterogeneity, we did not include studies specifically assessing treatment of HTN in persons with previous stroke.

The effect size of -0.049 (CI: -0.078 to -0.019 ) is small, but clinically important. Dementia affects approximately 45 million people worldwide, decreases self-esteem and quality of life, and increases healthcare costs and mortality. Current pharmacological management of dementia improves symptoms but does not address the aetiology or affect long term outcome. An intervention that addresses the aetiology of cognitive impairment and reduces cognitive decline will be of a large epidemiological significance. To improve the clinical interpretability of the results, we estimated the absolute magnitude of effect of aggressive treatment of HTN on reducing cognitive decline. We converted the pooled SMDs to an OR. Assuming linearity of effect and using the absolute rates of developing mild cognitive impairment or dementia in the control group from SPRINT-MIND, ${ }^{41}$ we estimated 16 mild cognitive impairment/probable dementia events would be prevented in 1000 patients followed for 10 years. This corresponded to an absolute risk reduction of $1.6 \%$ and an NNT of 63.

This systematic review has some limitations. First, the included studies had heterogeneity in BP goals and acquired $\mathrm{BP}$ at the end of the study. This was in part due to the change in HTN management guidelines which have reduced the BP goal over time. Second, the antihypertensives used in the included studies were different. We were unable to evaluate the effect size with different antihypertensives as the number of studies with a single class of antihypertensives was small. Third, included studies used different measures for assessing cognition. We used an SMD to circumvent this limitation. Fourth, the results of the meta-analysis are based on published randomized controlled trials (RCTs). As explanatory, non-pragmatic RCTs may not include the very old and frail patients; these results may not be generalisable to patients not adequately represented in these RCTs.

Despite these limitations, and the small effect size of reducing cognitive decline, this meta-analysis provides 


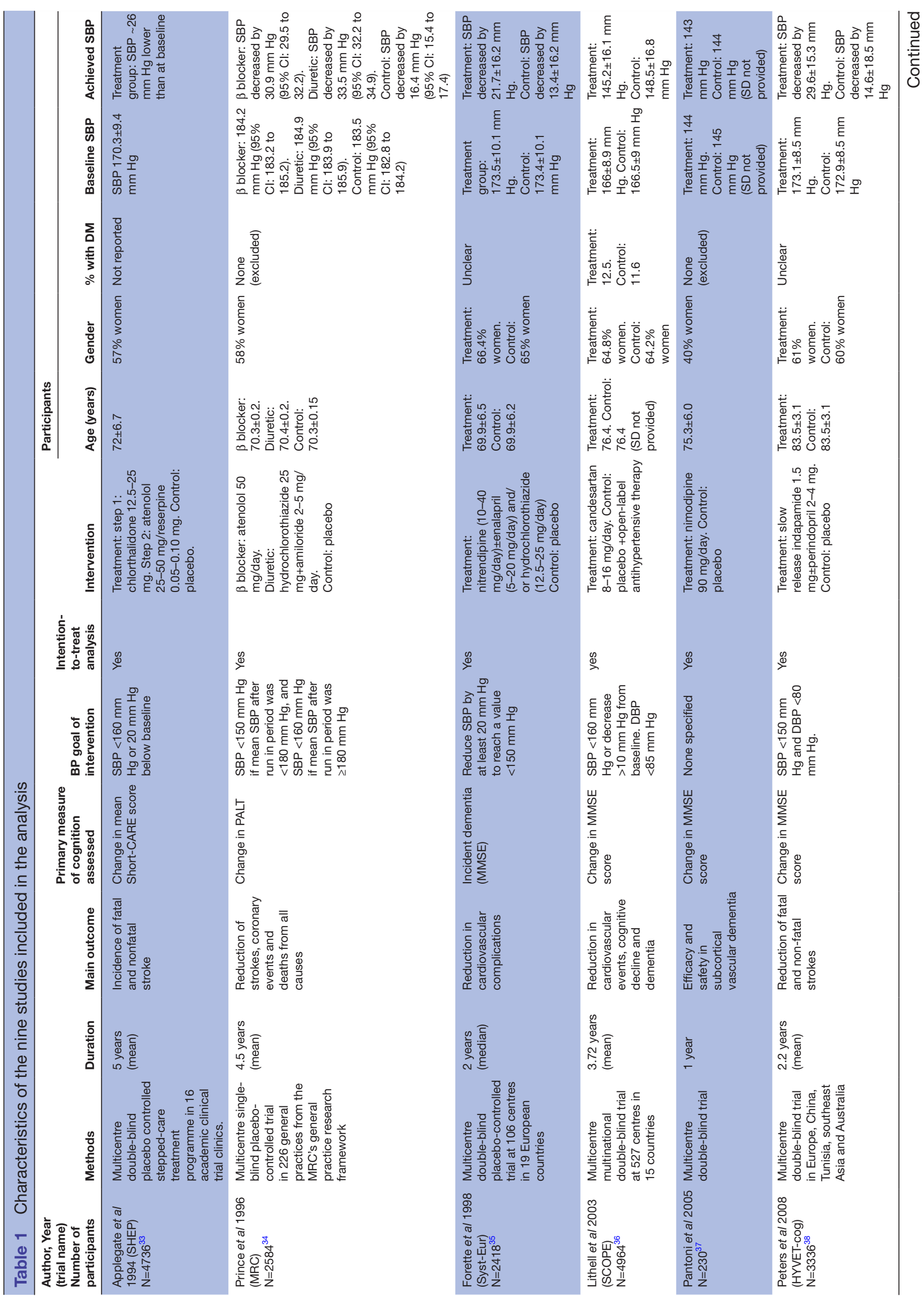




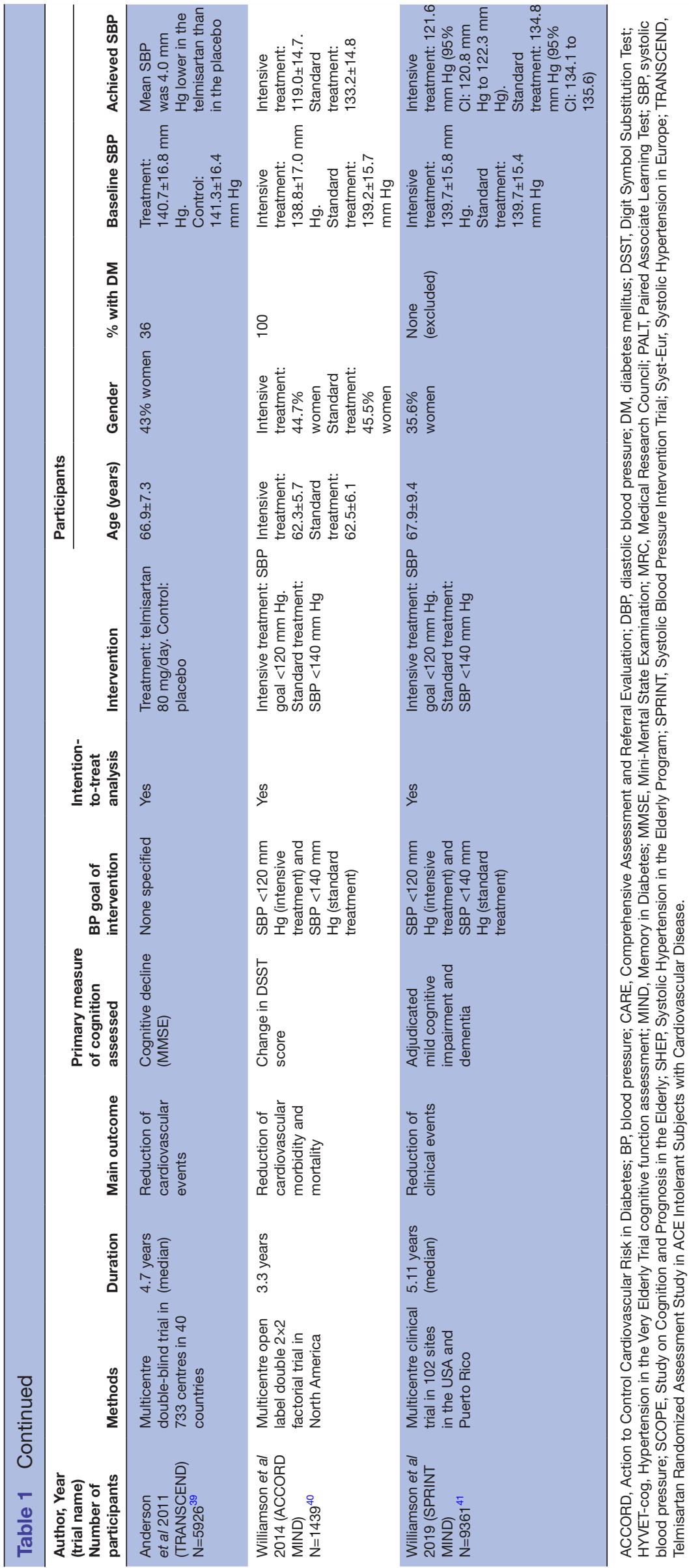




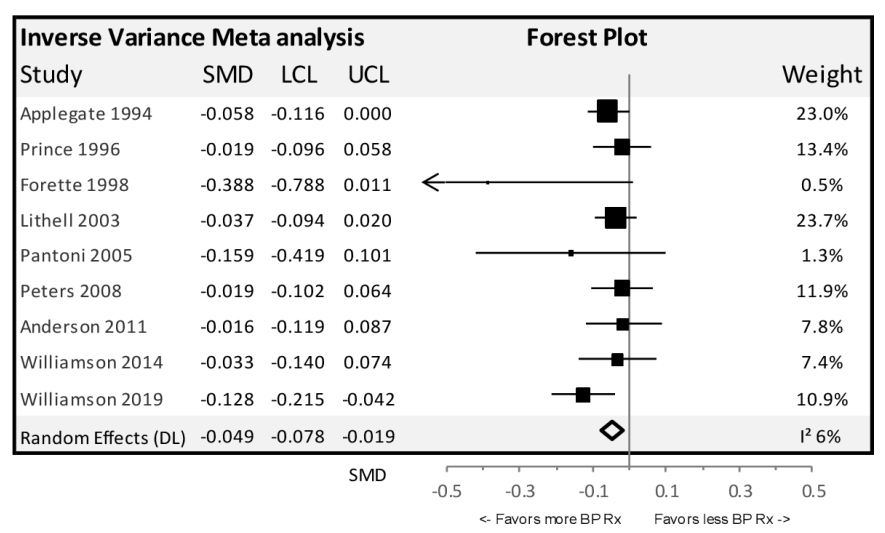

Figure 3 Forest plot with the effect on cognition (SMD with $95 \% \mathrm{Cl}$ ). BP, blood pressure; LCL, lower control limit; SMD, standardised mean difference; UCL, upper control limit.

important and clinically relevant information to guide HTN management. BP lowering decreases cardiovascular events and mortality. Despite these data, concern for worsening of cognition with BP lowering prevented clinicians from aggressive HTN management. This metaanalysis of published randomised controlled trials alleviates the concerns of adverse effects of treatment of HTN on cognitive decline.

The effect size of reducing cognitive decline was small. The benefit of HTN management may be currently underestimated due to the measure of cognition used in several studies. It is possible that lowering of BP affects certain domains of cognition more than the others and measurement of global cognition dissipated this effect. For example in SCOPE, ${ }^{36}$ the main study did not show any difference in MMSE scores, incident dementia or cognitive decline (defined by reduction in MMSE score by $\geq 4$ points from baseline between the active treatment and control groups), but a substudy of $\mathrm{SCOPE}^{46}$ showed an improvement in attention and episodic memory with treatment of HTN. Further studies with more extensive neuropsychological tests may be needed to prove this hypothesis.

In conclusion, treatment of HTN may be associated with a reduction in cognitive decline over time. Data from published RCTs show that treatment of HTN does not worsen cognition as previously feared. Although the effect of HTN treatment on reduction of cognitive decline is small, considering the high prevalence of dementia, the impact of dementia on quality of life and mortality, and the lack of promising pharmacological therapies, treating HTN to reduce cognitive decline may be a clinically relevant and effective intervention to reduce cognitive decline and dementia.

Contributors AG: conception or design of the work, data acquisition, analysis, interpretation of data and preparing the manuscript draft. SP: data acquisition, analysis, interpretation of data and preparing sections of the manuscript draft. SBi, SBe and JB: revised the manuscript critically for important intellectual content. GG: guided the study design, analysis, interpretation of data and critically revised the manuscript.

Funding NIH K23 AG055666 (AG).
Competing interests None declared.

Patient consent for publication Not required.

Provenance and peer review Not commissioned; externally peer reviewed.

Data availability statement Data are available upon reasonable request. All data relevant to the study are included in the article or uploaded as supplemental information. Additional data are available by emailing Aditi Gupta at agupta@kumc. edu.

Supplemental material This content has been supplied by the author(s). It has not been vetted by BMJ Publishing Group Limited (BMJ) and may not have been peer-reviewed. Any opinions or recommendations discussed are solely those of the author(s) and are not endorsed by BMJ. BMJ disclaims all liability and responsibility arising from any reliance placed on the content. Where the content includes any translated material, BMJ does not warrant the accuracy and reliability of the translations (including but not limited to local regulations, clinical guidelines, terminology, drug names and drug dosages), and is not responsible for any error and/or omissions arising from translation and adaptation or otherwise.

Open access This is an open access article distributed in accordance with the Creative Commons Attribution Non Commercial (CC BY-NC 4.0) license, which permits others to distribute, remix, adapt, build upon this work non-commercially, and license their derivative works on different terms, provided the original work is properly cited, appropriate credit is given, any changes made indicated, and the use is non-commercial. See: http://creativecommons.org/licenses/by-nc/4.0/.

ORCID iD

Aditi Gupta http://orcid.org/0000-0002-6286-802X

\section{REFERENCES}

1 GBD 2016 Dementia Collaborators. Global, regional, and national burden of Alzheimer's disease and other dementias, 1990-2016: a systematic analysis for the global burden of disease study 2016. Lancet Neurol 2019;18:88-106.

2 Prince M, Bryce R, Albanese E, et al. The global prevalence of dementia: a systematic review and metaanalysis. Alzheimers Dement 2013;9:63-75. e2.

3 Barnes DE, Yaffe K. The projected effect of risk factor reduction on Alzheimer's disease prevalence. Lancet Neurol 2011:10:819-28.

4 Mills KT, Bundy JD, Kelly TN, et al. Global disparities of hypertension prevalence and control: a systematic analysis of population-based studies from 90 countries. Circulation 2016;134:441-50.

5 Qiu C, Winblad B, Fratiglioni L. The age-dependent relation of blood pressure to cognitive function and dementia. Lancet Neurol 2005:4:487-99.

6 Sharp SI, Aarsland D, Day S, et al. Hypertension is a potential risk factor for vascular dementia: systematic review. Int J Geriatr Psychiatry 2011;26:661-9.

7 Gottesman RF, Albert MS, Alonso A, et al. Associations between midlife vascular risk factors and 25-year incident dementia in the Atherosclerosis risk in communities (ARIC) cohort. JAMA Neurol 2017;74:1246-54.

8 Elias MF, Wolf PA, D'Agostino RB, et al. Untreated blood pressure level is inversely related to cognitive functioning: the Framingham study. Am J Epidemiol 1993;138:353-64.

9 Kaess BM, Rong J, Larson MG, et al. Aortic stiffness, blood pressure progression, and incident hypertension. JAMA 2012;308:875-81.

10 Jefferson AL, Cambronero FE, Liu D, et al. Higher aortic stiffness is related to lower cerebral blood flow and preserved cerebrovascular reactivity in older adults. Circulation 2018;138:1951-62.

11 Rosenblum WI. Fibrinoid necrosis of small brain arteries and arterioles and miliary aneurysms as causes of hypertensive hemorrhage: a critical reappraisal. Acta Neuropathol 2008;116:361-9.

12 DuBose LE, Boles Ponto LL, Moser DJ, et al. Higher aortic stiffness is associated with lower global cerebrovascular reserve among older humans. Hypertension 2018;72:476-82.

13 de Leeuw F-E, de Groot JC, Oudkerk M, et al. Hypertension and cerebral white matter lesions in a prospective cohort study. Brain 2002;125:765-72.

14 Flint AC, Conell C, Ren X, et al. Effect of systolic and diastolic blood pressure on cardiovascular outcomes. N Engl J Med 2019;381:243-51.

15 Kuźma E, Lourida I, Moore SF, et al. Stroke and dementia risk: a systematic review and meta-analysis. Alzheimers Dement 2018;14:1416-26. 
16 Akoudad S, Wolters FJ, Viswanathan A, et al. Association of cerebral microbleeds with cognitive decline and dementia. JAMA Neurol 2016;73:934-43.

17 Whelton PK, Carey RM, Aronow WS, et al. 2017 ACC/AHA/AAPA ABC/ACPM/AGS/APhA/ASH/ASPC/NMA/PCNA guideline for the prevention, detection, evaluation, and management of high blood pressure in adults: a report of the American College of Cardiology/ American heart association Task force on clinical practice guidelines. Hypertension 2018;71:e13-115.

18 Khatib R, Schwalm J-D, Yusuf S, et al. Patient and healthcare provider barriers to hypertension awareness, treatment and follow up: a systematic review and meta-analysis of qualitative and quantitative studies. PLoS One 2014;9:e84238.

19 Graham DI. Ischaemic brain damage of cerebral perfusion failure type after treatment of severe hypertension. Br Med J 1975;4:739.

20 Hankey GJ, Gubbay SS. Focal cerebral ischaemia and infarction due to antihypertensive therapy. Med J Aust 1987;146:412-4.

21 Jansen PA, Gribnau FW, Schulte BP, et al. Contribution of inappropriate treatment for hypertension to pathogenesis of stroke in the elderly. Br Med J 1986;293:914-7.

22 Strandgaard S. Cerebral blood flow in the elderly: impact of hypertension and antihypertensive treatment. Cardiovasc Drugs Ther 1991;4 Suppl 6:1217-21.

23 Zhang R, Witkowski S, Fu Q, et al. Cerebral hemodynamics after short- and long-term reduction in blood pressure in mild and moderate hypertension. Hypertension 2007;49:1149-55.

24 Croall ID, Tozer DJ, Moynihan B, et al. Effect of standard vs intensive blood pressure control on cerebral blood flow in small vessel disease: the preserve randomized clinical trial. JAMA Neurol 2018;75:720-7.

25 Staessen JA, Richart T, Birkenhäger WH. Less atherosclerosis and lower blood pressure for a meaningful life perspective with more brain. Hypertension 2007;49:389-400.

26 Staessen JA, Thijs L, Richart T, et al. Placebo-Controlled trials of blood pressure-lowering therapies for primary prevention of dementia. Hypertension 2011;57:e6-7.

27 Igase M, Kohara K, Miki T. The association between hypertension and dementia in the elderly. Int J Hypertens 2012;2012:1-6.

28 McGuinness B, Todd S, Passmore P, et al. Blood pressure lowering in patients without prior cerebrovascular disease for prevention of cognitive impairment and dementia. Cochrane Database Syst Rev 2009;4:Cd004034.

29 Hasselblad V, Hedges LV. Meta-Analysis of screening and diagnostic tests. Psychol Bull 1995;117:167-78.

30 da Costa BR, Rutjes AWS, Johnston BC, et al. Methods to convert continuous outcomes into odds ratios of treatment response and numbers needed to treat: meta-epidemiological study. Int $J$ Epidemiol 2012;41:1445-59.

31 Lin L, Chu H, Murad MH, et al. Empirical comparison of publication bias tests in meta-analysis. J Gen Intern Med 2018;33:1260-7.

32 Borenstein M, Hedges L, Higgins J, et al. Comprehensive metaanalysis version 3, 2013. Available: https://www.meta-analysis.com/
33 Applegate WB, Pressel S, Wittes J, et al. Impact of the treatment of isolated systolic hypertension on behavioral variables. results from the systolic hypertension in the elderly program. Arch Intern Med 1994;154:2154.

34 Prince MJ, Bird AS, Blizard RA, et al. Is the cognitive function of older patients affected by antihypertensive treatment? results from 54 months of the medical Research Council's trial of hypertension in older adults. BMJ 1996;312:801-5

35 Forette F, Seux ML, Staessen JA, et al. Prevention of dementia in randomised double-blind placebo-controlled systolic hypertension in Europe (Syst-Eur) trial. Lancet 1998;352:1347-51.

36 Lithell H, Hansson L, Skoog I, et al. The study on cognition and prognosis in the elderly (scope). J Hypertens 2003;21:875-86.

37 Pantoni L, del Ser T, Soglian AG, et al. Efficacy and safety of nimodipine in subcortical vascular dementia: a randomized placebocontrolled trial. Stroke 2005;36:619-24.

38 Peters R, Beckett N, Forette F, et al. Incident dementia and blood pressure lowering in the hypertension in the very elderly trial cognitive function assessment (HYVET-COG): a double-blind, placebo controlled trial. Lancet Neurol 2008;7:683-9.

39 Anderson C, Teo K, Gao P, et al. Renin-Angiotensin system blockade and cognitive function in patients at high risk of cardiovascular disease: analysis of data from the ONTARGET and TRANSCEND studies. Lancet Neurol 2011;10:43-53.

40 Williamson JD, Launer LJ, Bryan RN, et al. Cognitive function and brain structure in persons with type 2 diabetes mellitus after intensive lowering of blood pressure and lipid levels: a randomized clinical trial. JAMA Intern Med 2014;174:324-33.

41 SPRINT MIND Investigators for the SPRINT Research Group, Williamson JD, Pajewski NM, et al. Effect of intensive vs standard blood pressure control on probable dementia: a randomized clinical trial. JAMA 2019;321:553-61.

42 Duval S, Tweedie R. Trim and fill: a simple funnel-plot-based method of testing and adjusting for publication bias in meta-analysis. Biometrics 2000;56:455-63.

43 Weiss J, Freeman M, Low A, et al. Benefits and harms of intensive blood pressure treatment in adults aged 60 years or older: a systematic review and meta-analysis. Ann Intern Med 2017;166:419-29.

44 Hughes D, Judge C, Murphy R, et al. Association of blood pressure lowering with incident dementia or cognitive impairment: a systematic review and meta-analysis. JAMA 2020;323:1934-44.

45 Ding J, Davis-Plourde KL, Sedaghat S, et al. Antihypertensive medications and risk for incident dementia and Alzheimer's disease: a meta-analysis of individual participant data from prospective cohort studies. Lancet Neurol 2020;19:61-70.

46 Saxby BK, Harrington F, Wesnes KA, et al. Candesartan and cognitive decline in older patients with hypertension: a substudy of the scope trial. Neurology 2008;70:1858-66. 